\title{
FACTORES ASOCIADOS A COMPLICACIONES PREQUIRÚRGICAS EN PACIENTES CON EQUINOCOCOSIS QUÍSTICA DE ÁREAS ENDÉMICAS DEL PERÚ
}

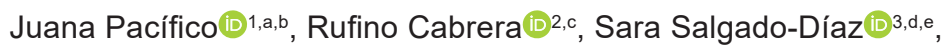 \\ Wilfredo Mormontoy (iD), Luis Suárez-Ognio (iD),d,g \\ 1 Departamento de Estadística, Demografía, Humanidades y Ciencias Sociales, Facultad de Ciencias y Filosofía, Universidad \\ Peruana Cayetano Heredia, Lima, Perú. \\ 2 Escuela de Medicina, Facultad de Ciencias de la Salud, Universidad Peruana de Ciencias Aplicadas, Lima, Perú. \\ 3 Servicio de Cardiología, Hospital Militar Central «Luis Arias Schereiber», Lima, Perú. \\ ${ }^{4}$ Departamento de Estadística, Demografía, Humanidades y Ciencias Sociales, Facultad de Ciencias y Filosofía, Universidad \\ Peruana Cayetano Heredia, Lima, Perú. \\ a Química farmacéutica; ${ }^{\mathrm{b}}$ maestra/o en Epidemiología; ${ }^{\mathrm{a}}$ biólogo; ${ }^{\mathrm{d}}$ médica/o cirujana/o; especialista en Cardiología; \\ f maestro en Salud Pública; ${ }^{\mathrm{g}}$ especialista en Epidemiología de Campo. \\ El presente estudio forma parte de la tesis de Pacífico J. Factores de riesgo para desarrollar complicaciones preoperatorias en \\ pacientes con equinococosis quística en un área endémica de los Andes centrales del Perú [tesis de especialidad en Estadística \\ para Investigación]. Lima: Facultad de Ciencias y Filosofía, Universidad Peruana Cayetano Heredia; 2020.
}

\section{RESUMEN}

Objetivo: Identificar los factores asociados a complicaciones prequirúrgicas en pacientes con equinococosis quística (EC) en áreas endémicas del Perú. Materiales y métodos: Se diseñó un estudio de casos $(n=43)$ y controles $(\mathrm{n}=127)$, sin emparejamiento, de fuente secundaria con una relación de caso-control de 1:3. Se definió como «caso» al paciente con EC que presentó complicaciones prequirúrgicas relacionadas o no con la enfermedad y el control sin complicación prequirúrgica. La variable respuesta fue complicación prequirúrgica y las de exposición fueron edad, género, grado de instrucción, ocupación, número de quistes, tamaño (diámetro en centímetros) y localización del quiste. Para identificar los factores de riesgo, primero se calcularon los odds ratio crudos $(\mathrm{ORc})$ mediante regresión logística; luego las variables que mostraron asociación significativa se ajustaron mediante análisis multivariado de regresión logística utilizando Stata ${ }^{\circledR}$ versión 14, y se obtuvieron los odds ratio ajustados (ORa). Resultados: El 55,8\% (24/43) de los casos fueron mujeres y la mediana de edad fue 35 años. Entre los controles, el 59,1\% (75/127) fueron mujeres y la mediana de edad fue 27 años. En el análisis de regresión logística multivariado, se encontró que tener más de 56 años tiene un ORa de 2,91 (intervalo de confianza [IC] 95\%: 1,01-8,43) y tener $\geq 3$ quistes tiene un ORa de 4,26 (IC 95\%: 1,62-11,22) para presentar complicaciones prequirúrgicas en pacientes con EQ. Conclusión: Los factores asociados para presentar complicaciones prequirúrgicas en $\mathrm{EQ}$ fueron el tener más de 56 años y el tener $\geq 3$ quistes.

Palabras clave: Equinococosis; Factores de Riesgo; Estudios de Casos y Controles; Enfermedades Desatendidas; Perú (fuente: DeCS BIREME).

\section{FACTORS ASSOCIATED WITH PRE-SURGICAL COMPLICATIONS IN PATIENTS WITH CYSTIC ECHINOCOCCOSIS FROM ENDEMIC AREAS OF PERU}

Citar como: Pacífico J, Cabrera R, Salgado-Díaz S, Mormontoy W, Suárez-Ognio L. Factores asociados a complicaciones prequirúrgicas en pacientes con equinococosis quística de áreas endémicas del Perú. Rev Peru Med Exp Salud Publica. 2021;38(1):3340. doi: https://doi.org/10.17843/ rpmesp.2021.381.5295.

Correspondencia: Juana Pacífico; Jr. Puno N.o 1002, Lima, Perú; juana. pacifico@unmsm.edu.pe

Recibido: $25 / 02 / 2020$

Aprobado: 23/09/2020

En línea: $04 / 01 / 2021$

\begin{abstract}
Objective: To identify risk factors to pre-surgical complications in patients with cystic echinococcosis (CE) in endemic areas of Peru. Materials and methods: Study of cases $(n=43)$ and controls $(n=127)$ not paired was designed, with a case-control ratio of 1:3 based on a secondary source. A case was defined as a patient with $\mathrm{CE}$ who presented pre-surgical complications related or not with the disease and the control without pre-surgical complication. The response variable was preoperative complication and the exposure variables were age, gender, degree of instruction, occupation, number, size (diameter in centimeters) and location of the cyst. To identify the risk factors, a bivariate logistic regression analysis was performed to obtain the crude odds ratios (cOR) and then the variables with significant association were adjusted by a multivariate logistic regression analysis using the Stata ${ }^{\circ}$ version 14 software, obtaining the adjusted odds ratios (aOR). Results: Fifty eight point eight percent (24/43) of the cases were women and the median age was 35 years and 59.1\% (75/127) of the controls correspond to the female gender and the median age was 27 years. The multivariate logistic regression analysis found that being over 56 years old has an aOR 2.91 (95\% confidence interval [CI]: 1.007-8.433) and having $\geq 3$ cysts has an aOR 4.26 (95\% CI: 1.62-11.22) for present pre-surgical complications in patients with CE. Conclusion: It was identified that being over 56 years old and having $\geq 3$ cysts are risk factors for presenting pre-surgical complications in CE.
\end{abstract}

Keywords: Echinococcosis; Risk Factors; Case-Control Studies; Neglected Diseases; Peru (source: MeSH NLM). 


\section{INTRODUCCIÓN}

La equinococosis quística (EQ), también conocida como hidatidosis, es una zoonosis parasitaria causada por la larva (metacéstode) de Echinococcus granulosus, un helminto parásito que en su forma adulta se encuentra en el intestino del perro y en otros cánidos ${ }^{(1)}$. La EQ suele ser asintomática, el cuadro clínico es variable y depende de factores que se relacionan tanto del huésped como del parásito ${ }^{(2)}$.

La EQ afecta a más de un millón de personas por año ${ }^{(2)} \mathrm{y}$ ocasiona 1009662 años de vida ajustados por discapacidad (AVAD) debido a la carga humana ${ }^{(3)}$. En el Perú, el costo estimado anual de la EQ humana es de USD 2420348 (IC 95\%: $1118384-4812722)^{(4)}$. En cinco países en las Américas, entre 2009 y 2014, Perú concentró el 70,3\% (20 785) del total de casos con una tasa de incidencia acumulada anual (TIA) entre 10 a 12,5 por cada 100000 habitantes ${ }^{(5)}$.

Por otro lado, la EQ ha sido reconocida por la Organización Mundial de la Salud (OMS) como una de las 17 enfermedades tropicales desatendidas ${ }^{(6)} \mathrm{y}$ recomendó validar las estrategias de control de equinococosis, mediante proyectos piloto, en países donde ha sido considerada como un problema de salud pública ${ }^{(7)}$. Actualmente, ya se disponen de herramientas de control para utilizarse a gran escala ${ }^{(7)}$.

El tratamiento percutáneo, la cirugía convencional, el uso de drogas antiparasitarias y el seguimiento u observación son de consenso internacional ${ }^{(8)}$; sin embargo, una considerable proporción de pacientes presentan complicaciones que requieren atención protocolizada ${ }^{(3)}$. Las complicaciones pueden ser por ruptura dentro o fuera del órgano comprometido, superinfección de los quistes, reacciones de anafilaxia a los antígenos del parásito, entre otros ${ }^{(8)}$. Se pueden detectar en la etapa prequirúrgica como posquirúrgica, la más común, es la fístula cistobiliar en localización hepática e incrementa, significativamente, la estancia hospitalaria posquirúrgica ${ }^{(9)}$.

En una serie de EQ del Perú, el 4\% presentó complicaciones prequirúrgicas y el 10,8\% posquirúrgicas. En las primeras, la desnutrición, el abdomen agudo y el empiema fueron las complicaciones más frecuentes ${ }^{(10)}$. Se sabe que la morbilidad en la hidatidosis hepática puede llegar hasta $80 \%$ y la letalidad hasta $10 \%$, y que estarían asociados a complicaciones de la enfermedad y a los procedimientos quirúrgicos ${ }^{(11)}$.

La edad, la localización hepática, el tamaño, la cantidad de quistes, la ruptura y la fístula biliar han sido identificados como factores o predictores de riesgo de las complicaciones o morbilidades posoperatorias ${ }^{(12-19)}$. También se han investigado predictores de mortalidad ${ }^{(12)}$, factores de riesgo de complicaciones durante la cirugía ${ }^{(20)}$ y asociados a la perforación peritoneal ${ }^{(21,22)}$, pero no se han realizado estudios orientados a identificar los factores de riesgo para desarrollar complicaciones prequirúrgicas. Su identificación es muy importante para reducir los costos de hospitalización y el riesgo de morir.

\section{MENSAJES CLAVE}

Motivación para realizar el estudio: La mayoría de las personas con equinococosis quística (EQ) o hidatidosis, ocasionada por larvas de la tenia del perro denominada Echinococcus granulosus, no desarrollan síntomas; sin embargo, una proporción de ellos se complican antes y/o después de la cirugía

Principales hallazgos: En este estudio, se encontró que ser mayor de 56 añosy tener $\geq 3$ quistes se asocian con complicaciones prequirúrgicas en los pacientes con $\mathrm{EQ}$.

Implicancias: Los resultados son de interés para la toma de decisiones antes de someter un paciente a cirugía.

Las técnicas quirúrgicas se han asociado al riesgo de morir ${ }^{(11-12)}$ y se han reportado hasta $20 \%$ de complicaciones en pacientes intervenidos quirúrgicamente ${ }^{(22)}$, por ello, consideramos pertinente estudiar los factores asociados a complicaciones prequirúrgicas.

El objetivo de este estudio fue identificar los factores asociados a complicaciones prequirúrgicas en pacientes con EQ procedentes de áreas endémicas de los Andes centrales del Perú.

\section{MATERIALES Y MÉTODOS}

\section{Diseño del estudio}

Este artículo se redactó siguiendo las recomendaciones de STROBE para estudios observacionales ${ }^{(23)}$. Se diseñó un estudio de casos y controles, sin emparejamiento con una relación de caso-control de 1:3 según una fuente secundaria. Se definió como «caso» al paciente con EQ que presentó complicaciones prequirúrgicas relacionadas o no con la enfermedad en el estudio original (empiema, insuficiencia respiratoria, neumonía, hemoptisis, atelectasia, neumotórax, abdomen agudo, colecistitis litiásica crónica, compromisos cardiacos, desnutrición, choque anafiláctico, hipertensión portal, sepsis, diabetes tipo II, epilepsia, obesidad y gestación) ${ }^{(10)}$. Se definió como «control» al paciente con $\mathrm{EQ}$ sin ninguna complicación prequirúrgica. Se han comparado retrospectivamente las variables de exposición entre los casos y los controles para identificar los que se asocian a complicaciones prequirúrgicas.

\section{Población en estudio}

Este estudio de fuente secundaria utilizó la base de datos de una serie de casos de EQ de los Andes centrales del Perú ${ }^{(10)}$. El 85,6\% de los casos procedían del departamento de Junín, el 8,4\% de Huancavelica, el 3,4\% de Pasco, el 1,3\% de Lima y el $0,5 \%$ de Huánuco ${ }^{(10)}$. 
Se incluyeron todos los casos y controles de la base del estudio original que tenían información completa de los potenciales factores de riesgo para desarrollar complicaciones prequirúrgicas.

\section{Variables}

La variable respuesta de tipo dicotómica (sí, no) fue complicación prequirúrgica, recogida de acuerdo con la definición descrita arriba ${ }^{(10)}$ y las variables de exposición fueron edad, género, grado de instrucción y ocupación y las clínicas: número de quistes, tamaño del quiste (diámetro) y localización del quiste (hígado y otra - pulmón, abdomen y otros-).

\section{Tamaño de muestra}

El tamaño de muestra para el diseño de caso-control sin emparejamiento se calculó utilizando el software Epi.Info versión 7.1.5.2 de los Centers for Disease Control and Prevention de los Estados Unidos de América. El cálculo del tamaño de muestra se realizó tomando en cuenta los siguientes parámetros: nivel de confianza al 95\%, potencia estadística del $80 \%$ y tres controles por cada caso, este último se utilizó para incrementar la eficiencia estadística ${ }^{(24)}$. Asimismo, se utilizó el tener dos o más quistes hidatídicos como variable de exposición, cuyos valores originales fueron redondeados, $54 \%$ entre los casos (con complicaciones prequirúrgicas), $30 \%$ entre los controles y un odds ratio (OR esperado) de 2,74 ${ }^{(14)}$. Se utilizó esta fuente debido a que no se encontró ningún estudio previo sobre factores de riesgo para desarrollar complicación prequirúrgica. El tamaño de muestra calculado fue 43 casos y 127 controles.

\section{Selección de la muestra}

Los controles ( $n=127$ ) se seleccionaron mediante un muestreo aleatorio simple realizado con el programa Stata versión 14 (StataCorp LLC), todos los controles seleccionados se verificaron para ver que tenían información completa y los que no tenían se reemplazaron aleatoriamente hasta completar la muestra (Figura 1). Se incluyeron 43 de 44 casos de la base de datos del estudio original ${ }^{(10)}$.

\section{Análisis estadístico}

A partir de la base de datos del estudio original, se creó una base de datos en Microsoft Excel con los casos y los controles seleccionados; luego, para su análisis se importaron a Stata versión 14 (StataCorp LLC).

El análisis exploratorio de las variables categóricas se realizó mediante frecuencias relativas y absolutas. Para mostrar el valor de p, se evaluaron los supuestos del uso de Chi cuadrado o prueba exacta de Fisher. La variable ocupación se midió como politómica y el grado de instrucción como una variable ordinal, que se recategorizó de ocho a cuatro categorías. En el caso de las variables continuas, se

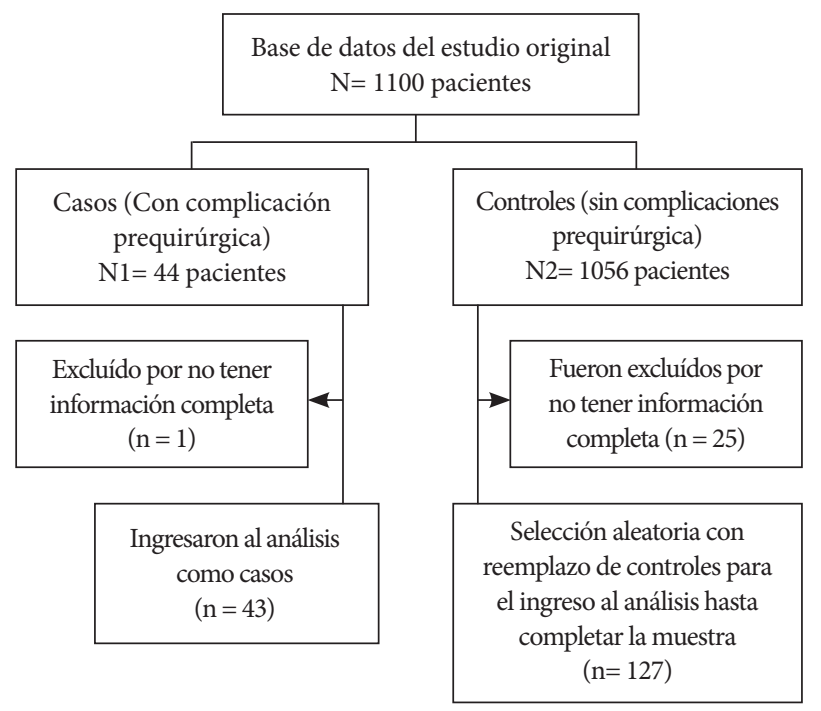

Figura 1. Flujograma de selección de los participantes del estudio.

evaluaron su normalidad mediante la prueba Shapiro Wilk y la homogeneidad de varianzas mediante el test de Levene. Cuando se demostró que las variables tenían una distribución no normal, se utilizó la prueba no paramétrica de suma de rangos de U. de Mann-Whitney (Wilcoxon o prueba de Wilcoxon-Mann-Whitney) para determinar el valor de $\mathrm{p}$, además, al tratarse de una distribución no normal se mostró el rango intercuartílico (RIQ) y la mediana.

Luego, las variables continuas como la edad de los pacientes, la cantidad y tamaño (diámetro) del quiste fueron categorizadas a una variable dicotómica. En el caso de edad, en China se ha reportado que la media de la edad de defunciones por EQ fue 56 años ${ }^{(25)}$ y consideramos que China como el Perú son países con prevalencias muy altas en el mundo ${ }^{(1)}$ que podrían tener características epidemiológicas similares. Asimismo, se fijó como punto de corte, 3 o más para el número de quistes de acuerdo con un estudio previo ${ }^{(13)}$ y $14 \mathrm{~cm}$ para el diámetro del quiste.

Luego, se realizó un análisis multivariado para identificar a las variables o factores que pudieran estar asociadas a complicaciones prequirúrgicas de manera independiente o controlando el efecto de las otras variables. Para ello, se aplicó el modelo de regresión logística multivariado y las variables que ingresaron al modelo fueron aquellas con un $\mathrm{p}<0,2$. Finalmente, se calcularon los OR ajustados (ORa) con un intervalo de confianza del $95 \%$.

Los sesgos se controlaron mediante la selección aleatoria de los controles, los criterios de inclusión de los casos y los controles, además del análisis de regresión multivariada de los potenciales factores de riesgo. En el análisis multivariado, el tamaño de quiste fue considerado como un potencial confusor. 


\section{Aspectos éticos}

Este estudio fue aprobado por el Comité Institucional de Ética en Investigación de la Universidad Peruana Cayetano Heredia y se contó con la autorización del Centro Nacional de Epidemiología, Prevención y Control de Enfermedades (CDC) del Ministerio de Salud para el uso de la base de datos.

\section{RESULTADOS}

Se incluyeron en el estudio 170 participantes, de los cuales, 43 fueron casos y 127 fueron controles. El 55,8\% (24) de los casos fueron del género femenino y la mediana de la edad fue 35 años, en cambio, el 59,1 \% (75) de los controles corresponden al género femenino y la mediana de edad fue 27 años. Otras características de los casos y de los controles se presentan en la Tabla 1.

En la Tabla 2, se muestran los resultados del análisis de regresión logística bivariado. Se encontró asociación entre tener más de 56 años, el grado de instrucción y tener $\geq 3$ quistes con el riesgo de desarrollar complicaciones prequirúrgicas en pacientes con $\mathrm{EQ}$, considerando un $\mathrm{p}<0,05$.

Tabla 1. Características de los casos (con complicaciones prequirúrgicas) y controles (sin complicaciones) de áreas endémicas de equinococosis quística.

\begin{tabular}{|c|c|c|c|c|c|}
\hline \multirow[t]{2}{*}{ Variable } & \multicolumn{2}{|c|}{$\begin{array}{l}\text { Casos (con complicaciones) } \\
((n=43)\end{array}$} & \multicolumn{2}{|c|}{$\begin{array}{l}\text { Controles (sin complicaciones) } \\
\qquad(\mathrm{n}=127)\end{array}$} & \multirow[t]{2}{*}{ Valor de $\mathrm{p}$} \\
\hline & $\mathbf{n}$ & $\%$ & $\mathbf{n}$ & $\%$ & \\
\hline \multicolumn{6}{|l|}{ Género } \\
\hline Femenino & 24 & 55,8 & 75 & 59,1 & $0,710^{*}$ \\
\hline Masculino & 19 & 44,2 & 52 & 40,9 & \\
\hline \multicolumn{6}{|l|}{ Edad (años) } \\
\hline Mediana (RIQ) & \multicolumn{2}{|c|}{$35(41)$} & \multicolumn{2}{|c|}{$27(27)$} & $0,457^{* *}$ \\
\hline Mayor a 56 años & 8 & 18,6 & 10 & 7,9 & $0,050^{* * *}$ \\
\hline Menor o igual a 56 años & 35 & 81,4 & 117 & 92,1 & \\
\hline \multicolumn{6}{|l|}{ Grado de instrucción } \\
\hline Superior & 4 & 9,3 & 17 & 13,4 & $0,419^{*}$ \\
\hline Secundaria & 12 & 27,9 & 49 & 38,6 & \\
\hline Preescolar y primaria & 25 & 58,1 & 57 & 44,8 & \\
\hline Sin grado de instrucción & 2 & 4,7 & 4 & 3,2 & \\
\hline \multicolumn{6}{|l|}{ Ocupación } \\
\hline Agricultor & 1 & 2,3 & 7 & 5,5 & $0,625^{*}$ \\
\hline Ama de casa & 8 & 18,6 & 30 & 23,6 & \\
\hline Estudiante & 20 & 46,5 & 62 & 48,8 & \\
\hline Otro & 14 & 32,6 & 28 & 22,0 & \\
\hline \multicolumn{6}{|l|}{ Tiempo de enfermedad (años) } \\
\hline Mediana (RIQ) & \multicolumn{2}{|c|}{$0,16(0,70)$} & \multicolumn{2}{|c|}{$0,25(0,60)$} & $0,912^{\star \star}$ \\
\hline \multicolumn{6}{|l|}{ Número de quistes } \\
\hline Mediana (RIQ) & \multicolumn{2}{|c|}{$1(2)$} & \multicolumn{2}{|c|}{$1(1)$} & $0,044^{* *}$ \\
\hline$\geq 3$ quistes & 11 & 25,6 & 10 & 7,9 & $0,002^{*}$ \\
\hline $1-2$ quistes & 32 & 74,4 & 117 & 92,1 & \\
\hline \multicolumn{6}{|l|}{ Tamaño del quiste (diámetro) } \\
\hline Mediana (RIQ) & \multicolumn{2}{|c|}{$10(4)$} & \multicolumn{2}{|c|}{$10(3)$} & $0,988^{* *}$ \\
\hline Mayor a $14 \mathrm{~cm}$ & 10 & 23,3 & 17 & 13,4 & $0,126^{*}$ \\
\hline Menor o igual a $14 \mathrm{~cm}$ & 33 & 76,7 & 110 & 86,6 & \\
\hline \multicolumn{6}{|l|}{ Localización del quiste } \\
\hline Más de una localización & 6 & 14,0 & 23 & 18,1 & $0,531^{*}$ \\
\hline Una localización & 37 & 86,0 & 104 & 81,9 & \\
\hline \multicolumn{6}{|l|}{ Órgano afectado } \\
\hline Hígado & 30 & 69,8 & 81 & 63,8 & $0,476^{*}$ \\
\hline Otro órgano & 13 & 30,2 & 46 & 36,2 & \\
\hline
\end{tabular}

'Variable de distribución no normal. ${ }^{\star}$ Prueba de chi cuadrado. ${ }^{* *}$ Suma de rangos de Wilcoxon. ${ }^{* *}$ Test exacto de Fisher.

RIQ: rango intercuartílico. 
Tabla 2. Análisis de regresión logística bivariada y multivariada para evaluar el riesgo de presentar complicaciones prequirúrgicas en pacientes con equinococosis quística.

\begin{tabular}{|c|c|c|c|c|}
\hline \multirow{2}{*}{ Variable } & \multicolumn{2}{|c|}{ Modelo crudo } & \multicolumn{2}{|c|}{ Modelo ajustados } \\
\hline & OR (IC 95\%) & Valor de p & ORa (IC 95\%) & Valor de $\mathrm{p}$ \\
\hline \multicolumn{5}{|l|}{ Género } \\
\hline Femenino & $1,14(0,56-2,29)$ & 0,710 & -- & \\
\hline Masculino & Referencia & & & \\
\hline \multicolumn{5}{|l|}{ Edad (años) } \\
\hline Mayor a 56 años & $2,67(0,98-7,29)$ & 0,050 & $3,05(1,07-8,64)$ & 0,035 \\
\hline Menor o igual a 56 años & Referencia & & Referencia & \\
\hline \multicolumn{5}{|l|}{ Grado de instrucción } \\
\hline Superior & Referencia & & & \\
\hline Secundaria & $1,04(0,29-3,66)$ & 0,950 & -- & \\
\hline Preescolar y primaria & $1,86(0,56-6,10)$ & 0,304 & -- & \\
\hline Sin grado de instrucción & $2,12(0,28-15,96)$ & 0,464 & -- & \\
\hline \multicolumn{5}{|l|}{ Ocupación } \\
\hline Agricultor & Referencia & & & \\
\hline Ama de casa & $1,86(0,19-17,45)$ & 0,584 & -- & \\
\hline Estudiante & $2,25(0,26-19,48)$ & 0,459 & -- & \\
\hline Otro & $3,5(0,39-31,31)$ & 0,262 & -- & \\
\hline \multicolumn{5}{|l|}{ Número de quistes } \\
\hline$\geq 3$ & $4,02(1,56-10,31)$ & 0,002 & $3,97(1,50-10,46)$ & 0,005 \\
\hline $1-2$ & Referencia & & Referencia & \\
\hline \multicolumn{5}{|c|}{ Tamaño del quiste (diámetro en $\mathrm{cm}$ ) } \\
\hline Mayor a 14 & $1,96(0,81-4,69)$ & 0,126 & $1,05(0,97-1,13)$ & 0,186 \\
\hline Menor o igual a 14 & Referencia & & Referencia & \\
\hline \multicolumn{5}{|l|}{ Localización del quiste } \\
\hline Más de una localización & $0,73(0,27-1,94)$ & 0,531 & -- & \\
\hline Una localización & Referencia & & & \\
\hline \multicolumn{5}{|l|}{ Órgano afectado } \\
\hline Hígado & $1,31(0,62-2,75)$ & 0,476 & --- & \\
\hline Otro órgano & Referencia & & & \\
\hline
\end{tabular}

Ajustado las variables mostradas en la columna. OR: odds ratio.

En la misma tabla, se muestran los resultados del análisis multivariado de regresión logística. Según la metodología descrita, ingresaron al modelo las variables con valor de $\mathrm{p}<0,200$. Se encontró que tener más de 56 años $\mathrm{y} \geq 3$ quistes son factores de riesgo para presentar complicaciones prequirúrgicas en pacientes con $\mathrm{EQ}$.

\section{DISCUSIÓN}

En este estudio de casos y controles sin emparejamiento, donde se evaluaron ocho variables como potenciales factores de riesgo para desarrollar complicaciones prequirúrgicas en pacientes con EQ de áreas endémicas de los andes centrales del Perú, utilizando un análisis multivariado de regresión logística, el tener más de 56 años y tener tres a más quistes hidatídicos fueron identificadas como factores de riesgo para complicaciones prequirúrgicas.

Este es el primer estudio para identificar factores de riesgo para complicaciones prequirúrgicas en pacientes con EQ, sobre la base de una de las series históricas de casos más grandes de EQ publicadas, procedentes de la sierra central del Perú ${ }^{(10)}$. La mayoría de los estudios en otros países endémicos ha servido para identificar los factores o predictores de riesgo para desarrollar complicaciones o morbili- 
dades posoperatorias ${ }^{(12,14-19)}$, predictores de mortalidad ${ }^{(12)}$, factores de riesgo durante la cirugía ${ }^{(20)}$ y factores asociados a la perforación peritoneal ${ }^{(21-22)}$. Es crucial identificar las complicaciones prequirúrgicas, porque permitiría una hospitalización oportuna, evitando complicaciones severas que podrían empeorar el pronóstico ${ }^{(21)}$ o permitiría intervenciones más seguras y apropiadas para reducir el riesgo de morir. Además, las técnicas quirúrgicas han sido identificadas como un factor asociado al riesgo de morir ${ }^{(11-12)}$ y la intervención quirúrgica en la identificación de los factores de riesgo para desarrollar las complicaciones posoperatorias en hidatidosis podría ser un sesgo.

En este estudio, se identificó que los pacientes con EQ mayores de 56 años tienen 3,05 veces la chance de presentar complicaciones prequirúrgicas en comparación con los que tienen 56 años o menos. En una serie histórica de muertes producidas por equinococosis en China, reportados entre 2008 y 2016, la media de la edad fue 56 años ${ }^{(25)}$. Por esa razón, tomamos como punto de corte para el análisis de la edad categorizada en dos estratos y también por las características epidemiológicas similares ${ }^{(1)}$. En un estudio de casos y controles en Chile, donde los autores no calcularon el tamaño de muestra, encontraron que los casos (con morbilidad posoperativa) que tenían un promedio de $59 \pm 18,8$ años, tenían un riesgo de 1,07 veces más de presentar la morbilidad en comparación con los controles que tenían un promedio de $41 \pm 16,3$ años ${ }^{(14)}$. En otro estudio longitudinal retrospectivo, a pesar de que la edad no se asoció a las complicaciones ni a la mortalidad, la media de edad de 491 pacientes con EQ fue 59,5 $\pm 20,4^{(22)}$.

En el presente estudio, la mediana de edad de los casos fue 35 con un RIQ de 41 años, mayor que la mediana y el RIQ de los controles (27 años). En un estudio sobre predictores de morbilidad y mortalidad en pacientes con EQ hepática, intervenidos quirúrgicamente, se encontró, que ser mayor de 40 años tiene un riesgo (OR) de 2,84 veces más comparado con los que tenían menos de 40 años y el riesgo se incrementaba con la edad ${ }^{(12)}$.

El tener más de 56 años como factor de riesgo para las complicaciones prequirúrgicas tiene plausibilidad, porque podría estar asociado a la exposición permanente al riesgo, es decir, a los huevos de E. granulosus, dado que los Andes centrales son áreas hiperendémicas con tasas de incidencias muy altas ${ }^{(5)}$. Por otro lado, se ha sugerido que en personas mayores, el desarrollo de complicaciones podría asociarse con las comorbilidades ${ }^{(15)}$, y también puede estar relacionado con el crecimiento de los quistes.

En el análisis bivariado de la edad categorizada, se encontró que se asoció en los estratos $\geq 40$ años y $<40$ años y entre $\geq 60$ años y $<60$ años, pero no se observó un incremento en la magnitud de la asociación. En España, el promedio de la edad en una cohorte retrospectiva de pacientes con complicaciones fue 59 años $\pm 20,3$ años ${ }^{(26)}$ y fue un factor de riesgo, lo que evidencia que el punto de corte que se tomó en este análisis tiene una base epidemiológica.

En este estudio, también se identificó que los pacientes con tres quistes a más tienen 3,97 veces más la chance de presentar complicaciones prequirúrgicas en comparación con los que tienen entre uno a dos quistes. Un mayor número de quistes aumentaría el riesgo de complicaciones posquirúrgicas de la $\mathrm{EQ}$, pero principalmente, de morir ${ }^{(12)}$. Los pacientes que tienen tres o más quistes tienen entre 2,55 hasta 3,1 veces más riesgo, que aquellos que tienen entre uno y dos quistes para desarrollar morbilidad o complicación posoperatoria $^{(13,16)}$. En una serie de casos en pacientes turcos, se encontró que el tener varios quistes y de tipo bilobular aumentaron significativamente el riesgo de la ruptura intrabiliar ${ }^{(20)}$. El tener tres o más quistes en los pacientes con EQ como factor de riesgo para presentar complicaciones prequirúrgicas puede deberse a múltiples exposiciones de las personas en áreas endémicas. En un estudio transversal, la edad media de los participantes evaluados en tres distritos de la región Junín y el tiempo de residencia mostraron una asociación estadística significativa para la presentación de un seropositivo para larvas de E. granulosus ${ }^{(27)}$. El Perú aún no tiene un programa nacional de control de EQ y las intervenciones han sido muy focalizadas.

En este artículo, el género, el grado de instrucción, la ocupación, el tamaño y la localización del quiste no se identificaron como factores de riesgo para presentar complicaciones prequirúrgicas; sin embargo, en un estudio previo, los pacientes con EQ hepática con localización centro hepática de la lesión principal de los quistes no complicados tienen 3,94 veces más riesgo comparados con los que tenían localización lateral para presentar morbilidad posoperatoria ${ }^{(14)}$. En una revisión sistemática, se identificó que los pacientes con hidatidosis hepática con quistes localizados en el «domo hepático» tienen 2,84 veces más riesgo que otras localizaciones hepáticas para desarrollar morbilidad posoperatoria ${ }^{(13)}$.

Por otro lado, en un estudio de cohorte prospectiva en el que se investigó si la comunicación quisto-biliar es un factor de riesgo para presentar morbilidad posoperatoria, los autores encontraron que los pacientes que tenían 15,4 $\pm 6,8 \mathrm{~cm}$ de diámetro tuvieron un riesgo relativo ( $R R$ ) de 1,49 veces más comparado con los que tenían quistes con diámetros menores; sin embargo, el riesgo fue mayor $(3,41)$ cuando se comparó el grupo sin comunicación quisto-biliar (CQB) y con dos o más episodios de CQB ${ }^{(14)}$. En una serie histórica, se encontró que los pacientes que tenían quistes $>10 \mathrm{~cm}$ de diámetro tuvieron una chance de 2,8 veces (OR) de presentar morbilidad o morir en comparación con aquellos pacientes que tenían $\leq 10 \mathrm{~cm}^{(12)}$.

En otro estudio en Turquía, al comparar los pacientes que tenían perforación peritoneal y sin perforación, los autores 
encontraron diferencias significativas en los pacientes que tenían quistes $>10 \mathrm{~cm}$ de diámetro frente a los que tenían diámetros menores del quiste ${ }^{(20)}$. En un estudio de caso-control se encontró que el tamaño del quiste se asoció al choque anafiláctico durante la cirugía con un OR de 3,028 (IC 95\%: 1,259-7,283) ${ }^{(19)}$. El no haber identificado que el tener quistes de $>14 \mathrm{~cm}$ de diámetro sea un factor de riesgo para desarrollar complicaciones prequirúrgicas podría deberse a las características de la edad de los casos, es decir, podría estar asociado al tamaño del quiste; sin embargo, se requieren mayores estudios, dado que actualmente las técnicas imagenológicas son de mayor capacidad resolutiva para detectar quistes pequeños o para tener mejor precisión respecto al diámetro de los quistes.

Este estudio aporta en la identificación de factores de riesgo para presentar complicaciones o morbilidad prequirúrgica lo que permitiría una hospitalización oportuna de los pacientes ${ }^{(21)}$, manejo apropiado y acceso al diagnóstico por imágenes. Se ha postulado que la cirugía en la práctica clínica se asocia frecuentemente a complicaciones, en los adultos mayores o en aquellos que tienen comorbilidad ${ }^{(26)}$. Asimismo, las complicaciones posoperatorias son una de las causas más comunes asociadas a la mortalidad ${ }^{(22)}$; por ello, la cistectomía no es considerada como un método de cura definitiva ${ }^{(28)}$. Las técnicas quirúrgicas convencionales tendrían un elevado riesgo de desarrollar morbilidad y mortalidad en los pacientes con equinococosis quística hepática ${ }^{(12)}$, así, las complicaciones como la anafilaxia, infección del quiste, fístula biliar y el absceso intraabdominal son frecuentes en los pacientes intervenidos quirúrgicamente ${ }^{(28-29)}$.

\section{REFERENCIAS BIBLIOGRÁFICAS}

1. Craig PS, McManus DP, Lightowlers MW, Chabalgoity JA, Garcia HH, Gavidia CM, et al. Prevention and control of cystic echinococcosis. Lancet Infect Dis. 2007;7(6):385-94. doi: 10.1016/S14733099(07)70134-2.

2. Agudelo Higuita NI, Brunetti E, McCloskey C. Cystic Echinococcosis. J Clin Microbiol. 2016;54(3):518-23. doi: 10.1128/JCM.02420-15.

3. Budke CM, Deplazes P, Torgerson PR. Global socioeconomic impact of cystic echinococcosis. Emerg Infect Dis. 2006;12(2):296-303. doi: 10.3201/eid1202.050499.

4. Moro PL, Budke CM, Schantz PM, Vasquez J, Santivañez SJ, Villavicencio J. Economic Impact of Cystic Echinococcosis in Peru. PLoS Negl Trop Dis. 2011;5(5): e1179. doi: 10.1371/journal.pntd.0001179.

5. Pavletic CF, Larrieu E, Guarnera EA, Casas N, Irabedra P, Ferreira C, et al. Cystic echinococcosis in South America: a call for action. Rev Panam Salud Publica. 2017;41:e42. doi: 10.26633/RPSP.2017.42.

6. World Health Organization (WHO). WHO Accelerating work to overcome the global impact of neglected tropical diseases - A roadmap for implementation [Internet]. WHO; 2012 [citado el 7 de enero de 2020]. Disponible en: https://apps.who.int/iris/bitstream/ handle/10665/70809/WHO_HTM_NTD_2012.1_eng.pdf.

7. Organización Panamericana, IRIS Repositorio institucional. Centro Panamericano de Fiebre Aftosa, Prevención y control de la Hidatidosis en el nivel local: iniciativa sudamericana para el control y vigilancia
Por ello, estudiar los factores asociados a complicaciones prequirúrgicas, puede ser más importantes y útiles que estudiar las complicaciones posoperatorias para reducir el riesgo de las complicaciones, muerte, estancia hospitalaria y el impacto económico en las familias.

El estudio tiene entre sus limitaciones la antigüedad de los datos, el uso de una fuente secundaria, la limitada caracterización de las complicaciones prequirúrgicas en el estudio original ${ }^{(10)}$, la inclusión de casos con complicaciones no necesariamente relacionadas a la enfermedad, el pequeño tamaño de muestra de los casos, y los sesgos asociados al diseño.

En conclusión, en este estudio de casos y controles se identificaron que el tener más de 56 años y tener tres o más quistes en pacientes con EQ son factores de riesgo que asocian para desarrollar complicaciones prequirúrgicas en pacientes provenientes de la sierra central del Perú.

Contribuciones de los autores: JP y RC participaron en la concepción y diseño del artículo, selección de los casos y los controles, el análisis e interpretación de datos. SSD participó en la recolección de los datos en el estudio original. WM participó en la asesoría del análisis estadístico, revisión de los borradores y aprobación de la versión de sometimiento. JP, RC, SSD y LSO participaron en la redacción del artículo, revisión crítica y aprobación de la versión de sometimiento.

Financiamiento: El estudio original de donde se obtuvo la base de datos fue financiado por el Centro Nacional de Epidemiología, Prevención y Control de Enfermedades (CDC), Ministerio de Salud.

Conflictos de interés: Los autores no tienen ningún conflicto de interés que declarar. de la equinococosis quística / hidatidosis [Internet]. OPS; 2017 [citado el 7 de julio de 2020]. Disponible en: https://iris.paho.org/ handle/10665.2/34173.

8. Vuitton DA, McManus DP, Rogan MT, Romig T, Gottstein B, Naidich $A$, et al. International consensus on terminology to be used in the field of echinococcoses. Parasite. 2020;27:41. doi: 10.1051/parasite/2020024.

9. Demircan O, Baymus M, Seydaoglu G, Akinoglu A, Sakman G. Occult cystobiliary communication presenting as postoperative biliary leakage after hydatid liver surgery: Are there significant preoperative clinical predictors?. Can J Surg. 2006;49(3):177-84.

10. Salgado DS, Suárez-Ognio L, Cabrera R. Características clínicas y epidemiológicas de la equinococosis quística registradas en un área endémica en los Andes centrales del Perú (1991-2002). Neotrop Helminthol. 2007;1(2):69-84

11. Manterola C, Otzen T. Surgical Alternatives Used in the Treatment of Liver Hydatid Cyst. A Systematized Approach Based on Evidence (an Overview). Int J Morphol. 2016;34(2):699-707. doi: 10.4067/S071795022016000200044.

12. Daradkeh S, EL-Muhtaseb H, Farah G, Sroujieh AS, Abu-Khalaf M. Predictors of morbidity and mortality in the surgical management of hydatid cyst of the liver. Langenbecks Arch Surg. 2007;392(1):35-9. doi: 10.1007/s00423-006-0064-2. 
13. Manterola C, Otzen T, Muñoz G, Alanis M, Kruuse E, Figueroa G. Cirugía de la hidatidosis hepática. Factores de riesgo y variables asociadas al desarrollo de morbilidad postoperatoria. Revisión global de la evidencia existente. Cir Esp. 2017;95(10):566-76. doi: 10.1016/j. ciresp.2017.08.009.

14. Manterola C, Otzen T, Urrutia S, MINCIR Group (Methodology and Research in Surgery). Risk factors of postoperative morbidity in patients with uncomplicated liver hydatid cyst. Int J Surg. 2014;12(7):6959. doi: 10.1016/j.ijsu.2014.05.063.

15. Manterola DC, Bustos ML, Vial GM, Moraga CJ, Grupo MINCIR. ¿Es la comunicación quisto-biliar, un factor de riesgo para el desarrollo de morbilidad postoperatoria en pacientes con hidatidosis hepática? Rev Chil Cir. 2009;61(3):229-35.

16. El Malki HO, El Mejdoubi Y, Souadka A, Mohsine R, Ifrine L, Abouqal $\mathrm{R}$, et al. Predictive factors of deep abdominal complications after operation for Hydatid cyst of the Liver: 15 years of experience with 672 patients. J Am Coll Surg. 2008;206(4):629-37. doi: 10.1016/j. jamcollsurg.2007.11.012.

17. Manterola C, Urrutia S, Grupo MINCIR. Morbilidad postoperatoria en pacientes con hidatidosis hepática complicada. Rev Chilena Infectol. 2015;32(1):43-9.

18. Jaén-Torrejimeno I, López-Guerra D, Prada-Villaverde A, Blanco-Fernández G. Pattern of Relapse in Hepatic Hydatidosis: Analysis of 238 Cases in a Single Hospital. J Gastrointest Surg. 2020;24(2):361-367. doi:10.1007/s11605-019-04163-7.

19. Khachatryan AS. Analysis of lethality in Echinococcal disease. Korean J Parasitol. 2017;55(5):549-53. doi: 10.3347/kjp.2017.55.5.549.

20. Akcan A, Akyildiz H, Artis T, Ozturk A, Deneme MA, Ok E, et al. Peritoneal perforation of liver hydatid cysts: clinical presentation, predisposing factors, and surgical outcome. World J Surg. 2007;31(6):1284-91. doi: 10.1007/s00268-007-9024-4.
21. Akcan A, Sozuer E, Akyildiz H, Ozturk A, Atalay A, Yilmaz Z. Predisposing factors and surgical outcome of complicated liver hydatid cysts. World J Gastroenterol. 2010;16(24):3040-8. doi: 10.3748/wjg. v16.i24.3040.

22. Velasco-Tirado V, Romero-Alegria A, Pardo-Lledías J, Alonso-Sardón M, Lopez-Bernus A, Quiñones Sampedro J, et al. Management of cystic echinococcosis in the last two decades: what have we learned?. Trans R Soc Trop Med Hyg. 2018;112(5):207-215. doi: 10.1093/trstmh/try050.

23. VandenbrouckeJP, von Elm E, Altman DG, Gøtzsche PC, Mulrow CD, Pocock SJ, et al.; STROBE Initiative. Strengthening the Reporting of Observational Studies in Epidemiology (STROBE): explanation and elaboration. Epidemiology 2007;18:805-35. doi: 10.1097/EDE.0b013e3181577511.

24. Soto A, Cvetkovich A. Estudios de casos y controles. Rev Fac Med Hum. (Lima). 2020;20(1):138-43. doi:10.25176/RFMH.v20i1.2555.

25. Tian T, Chun-Li C, Wei-Ping W, Tie-Wu J, Men-Bao Q, Yu-Wan H, et al. [Epidemiological characteristics of echinococcosis death cases in China from 2008 to 2016]. Zhongguo Xue Xi Chong Bing Fang Zhi Za Zhi. 2018;30(3):282-8.

26. Collado-Aliaga J, Romero-Alegría Á, Alonso-Sardón M, Muro A,López-Bemus A, Velasco-Tirado V, et al. Complications associated with initial clinical presentation of Cystic Echinococcosis: A 20-year cohort analysis. Am J Trop Med Hyg. 2019;101(3):628-635. doi: 10.4269/ajtmh.19-0019.

27. Santivañez SJ, Náquira C, Gavidia C, Tello L, Hernández E, Brunetti E, et al. Factores domiciliarios asociados con la presencia de hidatidosis humana en tres comunidades rurales de Junín, Perú. Rev Peru Med Exp Salud Publica. 2010; 27(4):498-505.

28. Pakala T, Molina M, Wu GY. Hepatic Echinococcal Cysts: A Review. J Clin Transl Hepatol. 2016;4(1):39-46. doi: 10.14218/JCTH.2015.00036.

29. Montúfar-Valer A, Huapaya-Jurado FL. Características clínicas, radiológicas y laboratoriales de pacientes con hidatidosis hepática en un hospital de referencia nacional, Lima 1997-2010. Rev Gastroenterol Perú. 2014;34(3):203-9. 